\title{
Shape of the northern hemisphere of Mars from the Mars Orbiter Laser Altimeter (MOLA)
}

\author{
Maria T. Zuber ${ }^{1,2}$, David E. Smith ${ }^{2}$, Roger J. Phillips ${ }^{3}$, Sean C. Solomon ${ }^{4}$, \\ W. Bruce Banerdt ${ }^{5}$, Gregory A. Neumann ${ }^{1,2}$, Oded Aharonson ${ }^{1}$
}

\begin{abstract}
Eighteen profiles of $\sim \mathrm{N}-\mathrm{S}$-trending topography from the Mars Orbiter Laser Altimeter (MOLA) are used to analyze the shape of Mars' northern hemisphere. MOLA observations show smaller northern hemisphere flattening than previously thought. The hypsometric distribution is narrowly peaked with $>20 \%$ of the surface lying within 200 $m$ of the mean elevation. Low elevation correlates with low surface roughness, but the elevation and roughness may reflect different mechanisms. Bouguer gravity indicates less variability in crustal thickness and/or lateral density structure than previously expected. The $3.1-\mathrm{km}$ offset between centers of mass and figure along the polar axis results in a pole-to-equator slope at all longitudes. The N-S slope distribution also shows a subtle longitude-dependent variation that may represent the antipodal effect of the formation of Tharsis.
\end{abstract}

\section{Introduction}

During the fall of 1997 the laser altimeter [Zuber et al., 1992] on the Mars Global Surveyor (MGS) spacecraft [Albee et al., 1998] acquired 18 profiles across the northern hemisphere of Mars. These tracks provided over 200,000 measurements of the radius of the planet between latitudes $82^{\circ}$ $\mathrm{N}$ and $12^{\circ} \mathrm{S}$, with a precision at the $30-\mathrm{cm}$ level and accuracy of $\sim 30 \mathrm{~m}$ [Smith et al., 1998]. The profiles were distributed approximately uniformly in longitude $\left(\sim 20^{\circ}\right.$ or $1200-\mathrm{km}$ separation at the equator), and provide new constraints on Mars' long-wavelength shape.

\section{Radius and Flattening}

MOLA observations have provided a new estimate for Mars' mean equatorial $(3396.0 \pm 0.3 \mathrm{~km})$ and north polar $(3373.4 \pm 0.5 \mathrm{~km})$ radii. The polar radius is based on extrapolation above $81^{\circ} \mathrm{N}$ and does not include the contribution of the polar deposits. The corresponding flattening of the northern hemisphere is $1 /(150 \pm 4)$, ignoring the possible effect of flexural loading by polar deposits [Solomon et al., 1998], which could depress the polar lithosphere and

\footnotetext{
${ }^{1}$ Department of Earth, Atmospheric, and Planetary Sciences, Massachusetts Institute of Technology, Cambridge

${ }^{2}$ Laboratory for Terrestrial Physics, NASA Goddard Space Flight Center, Greenbelt, Maryland

${ }^{3}$ Department of Earth and Planetary Sciences, Washington University, St. Louis, Missouri

${ }^{4}$ Department of Terrestrial Magnetism, Carnegie Institution of Washington, Washington, D.C.

${ }^{5}$ Jet Propulsion Laboratory, Pasadena, California

Copyright 1998 by the American Geophysical Union.
}

Paper number 1998GL900129.

0094-8276/98/1998GL900129\$05.00 potentially change the flattening by at most $5 \%$. Allowing for the 3.1-km offset of the center of mass (COM) from the center of figure (COF) along the polar axis [Smith and Zuber, 1996], we can expect the flattening of the full planet to be less than that of the northern hemisphere by $\sim 15 \%$, or $1 / 174 \pm 6$. This is significantly less than the earlier value of 1/154.4 [Bills and Ferrari, 1978] and slightly less than the ellipsoidal value of $1 / 166.53$ [Smith and Zuber, 1996] that have been used in geophysical analyses. However, it is larger than the flattening of $1 / 192$ used in making maps [Wu, 1991]. Consideration of ice cap topography will further decrease the value. The importance of a smaller flattening, if verified by future observations, is that it implies that Mars has less global-scale topography than previously thought, which will have implications for models of Mars' internal structure and dynamics.

\section{Hypsometric Properties}

The fundamental aspects of northern hemisphere shape are the low elevation relative to the older, heavily cratered southern hemisphere and the flatness and gentle tilting of the northern plains. Fig. 1a shows that the distribution of northern hemisphere elevations peaks sharply at $\sim-4.0$ $\mathrm{km}$ relative to the equipotential surface defined by Mars' equatorial radius. More than $12 \%$ of the sampled surface returns lie within $100 \mathrm{~m}$ of this level, and almost $21 \%$ are within $200 \mathrm{~m}$.

To understand the relationship between elevation and flatness, we compute the inter-quartile scale (IQS), the distance from the first to third quartiles of the slope distribution, scaled to unity for a normal distribution. This estimator is insensitive to outliers produced by local processes. In Fig. 1b a histogram of this roughness estimator is plotted for three subsets of the returns. The histograms were accumulated in bins $0.25^{\circ}$ wide and normalized individually to mitigate the current sampling bias. The highlands subset is the roughest $\left(\mathrm{IQS}>3^{\circ}\right.$ ), while the $85 \%$ of the surface lower than $2.5 \mathrm{~km}$ has a $10-\mathrm{km}$ slope variance $<0.25^{\circ}$ (Fig. 1c).

Statistical analysis of topography on planetary surfaces for which altimetry has been obtained suggests a depositional mechanism to explain the smoothest areas of the northern plains, because only known depositional surfaces on Earth have comparable roughness properties [Aharonson et al., 1998]. The low elevation of northern Mars is a prime reason why the concept of a hemispheric-scale ocean has been invoked [Parker et al., 1993]. While the correlation of low elevation and low surface roughness would be consistent with an origin by marine sedimentation, an ocean is not a unique interpretation of the observations. It is probable that the low elevation and low roughness have separate causes, the former by an endogeneic or exogeneic dynamical mechanism, and the latter due to subsequent surface modification. 

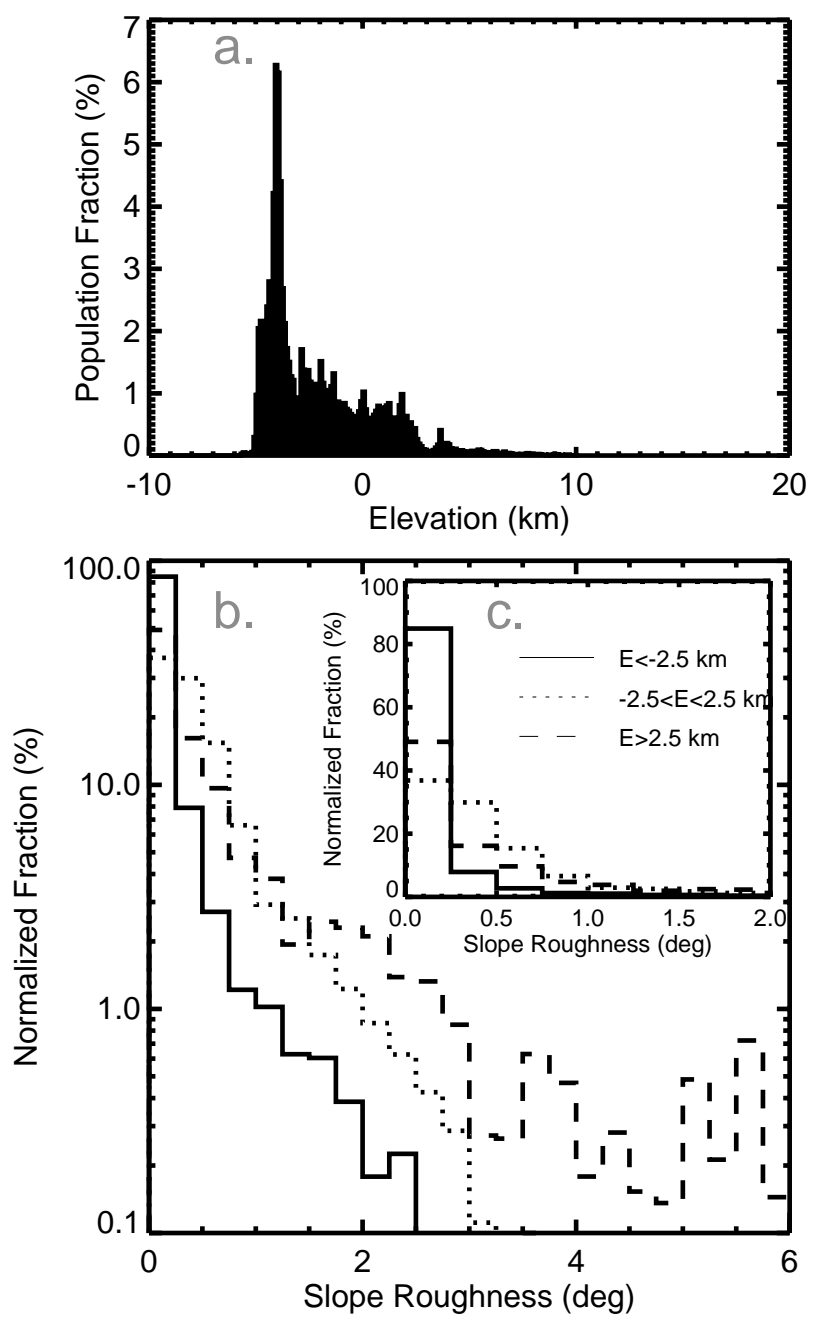

Figure 1. (a) Hypsogram of MOLA elevations in 100$\mathrm{m}$ wide bins. (b) Histogram of IQS of 10-km-scale slopes, divided into three elevation bins. (c) Low-roughness portion of (b), magnified by means of a linear vertical scale.

\section{Long-Wavelength Internal Structure}

The current quality of topography and gravity data combined with the lack of an independent observation of at least local subsurface structure preclude, at present, an inversion for crustal thickness. (In contrast, seismic data on the Moon have pegged crustal thickness within a small area to which thicknesses calculated elsewhere can be "anchored".) However, some aspects of the shallow subsurface density distribution can be addressed via calculation of Bouguer gravity, which is the surface gravity field that remains after correction for topography, and thus reflects the distribution of subsurface mass. Figure 2 shows contour maps of longwavelength free-air gravity, topography, and Bouguer gravity.

The gravity field is the GMM-1 model [Smith et al., 1993], which was derived from Doppler tracking observations of the Viking and Mariner 9 orbiters. In the model shown as the free-air gravity, we have removed $95 \%$ of the $C_{2,0}$ harmonic coefficient, which corresponds to the estimated hydrostatic contribution to the planetary flattening [Zuber and Smith,
1997]. The $5 \%$ of $C_{2,0}$ that we retain represents the approximate non-hydrostatic contribution of Tharsis topography and the flattening to the long-wavelength gravity field, as constrained by the observed moment of inertia [Folkner et al., 1997]. (Tharsis is a 10-km-high planet-scale feature that is centered nearly on the equator, and is not fully compensated. Thus it is not easily separable from Mars' topographic flattening.)

The topography model was derived from MOLA observations in the northern hemisphere and re-processed Viking and Mariner 9 occultation radii [Smith and Zuber, 1996] in the southern hemisphere. The topography model is the $12 \times 12$ component ( $\sim 900 \mathrm{~km}$ spatial resolution) of a $30 \times 30$ model derived from a combination of the altimetry and occultations, along with a power law constraint [Kaula, 1966] to control numerical instability. The topography model contains the degree 1 harmonic terms that correspond to the offsets of the geometric center of the planet from the COM along the polar axis and in the direction of Tharsis. The long-wavelength topographic control enables resolution of subtle large structures. For example, Fig. $2 \mathrm{~b}$ reveals a 1500-km circular depression with maximum depth of $2.5 \mathrm{~km}$ below its surroundings centered at lat $=45^{\circ} \mathrm{N}$, lon $=110^{\circ} \mathrm{E}$. The center of the depression is close to that of the proposed Utopia Basin [McGill, 1989], though that structure was hypothesized to be nearly twice the diameter of the structure resolved by MOLA. This feature also appears as a prominent mascon in gravity.

In the idealized case of a constant density crust, Bouguer gravity arises solely from topographic variations at the crust/mantle boundary that may or may not be isostatic. Under the assumption of local (Airy) isostasy, Bouguer anomalies can be interpreted in terms of crustal thickness. This interpretation neglects the possibility of lateral variations in crustal density or deeper mass variations. Lateral internal density variations would decrease the amplitude of crustal thickness variations implied by an Airy model. Fig. $2 \mathrm{c}$ would imply a gradual decrease in crustal thickness (or increase in crustal density) with increasing latitude outside Tharsis, but it could also indicate a constant density crust with progressively higher density material at greater depth as latitude increases. The variability of the crustal thickness under Airy isostasy associated with Fig. 2c is less than in previous long-wavelength inversions [cf. Kiefer et al., 1996]. An alternative interpretation is less variation in lateral subsurface density. Because the gravity field has not changed from that used in previous work, the reduction in variability is due to the narrow hypsometric distribution of northern hemisphere topography now revealed by MOLA altimetry.

\section{Long-Wavelength Slopes}

At the current MOLA accuracy, slopes of order $\pm 0.001^{\circ}$ can be resolved over wavelengths of $1800 \mathrm{~km}$. Subtle variations in long-wavelength topography have bearing on the processes that produce global physiography. Here we focus on slopes that can be resolved along approximate lines of longitude, because these can be analyzed with the current observations. In Mars' northern hemisphere, the COM/COF offset along the polar axis will cause a pole-to-equator upward slope of $\sim 0.036^{\circ}$ at all longitudes [Smith et al., 1998]. To investigate whether there are other identifiable longwavelength influences on the slope distribution, we have sub- 
(a)
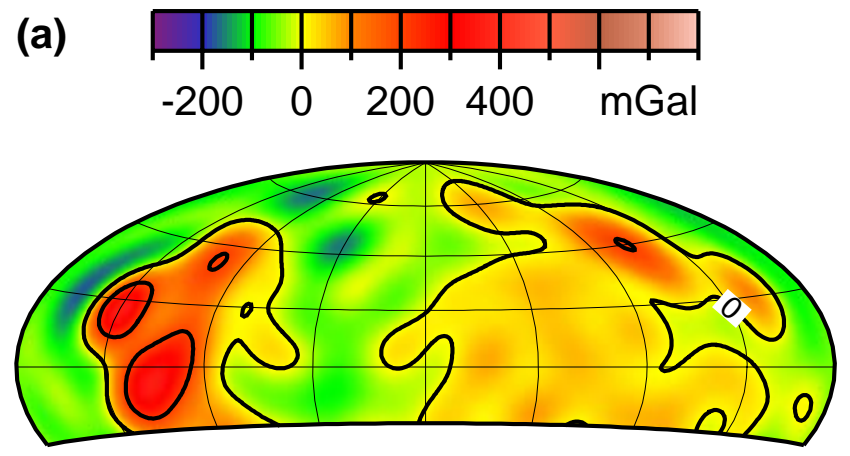

(b)
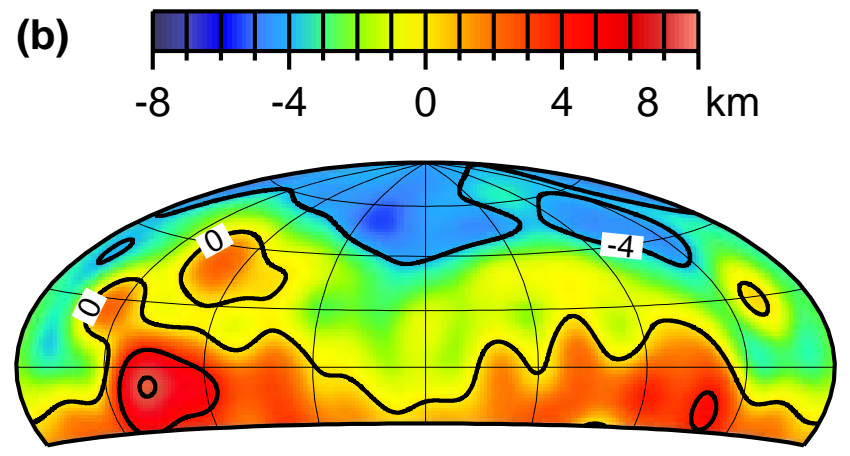

(c)
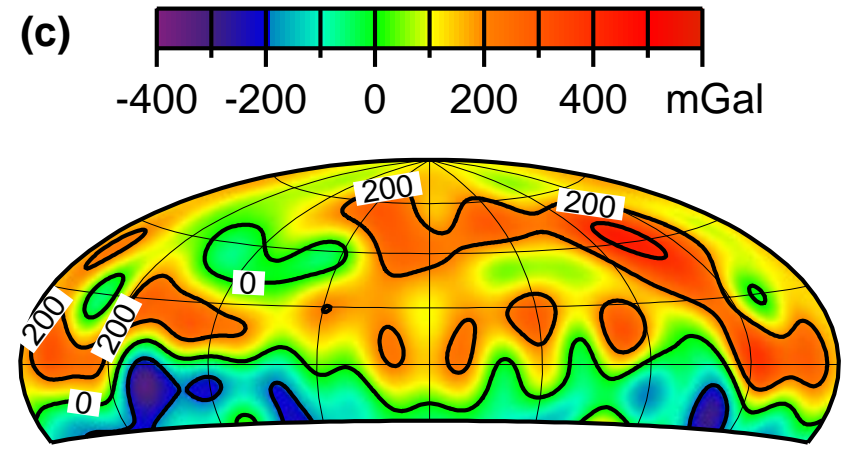

Figure 2. Twelfth degree and order models of Mars' (a) free-air gravity, (b) topography, and (c) Bouguer gravity. Zero longitude is the central vertical axis, the longitudinal line spacing is $45^{\circ}$, and the latitudinal line spacing is $22.5^{\circ}$. The maps are truncated at latitude $22.5^{\circ} \mathrm{S}$ because the only new data are from northern hemisphere altimetry. Tharsis is at the left-center of each map. The Bouguer anomaly map assumes a crustal density $\rho_{c}=2800 \mathrm{~kg} \mathrm{~m}^{-3}$.

tracted the COM/COF effect from the MOLA profiles. To isolate the slope signature associated with the low degree northern hemispheric shape, we excluded the topography of the Elysium province and structures associated with the dichotomy boundary. Figure 3 shows a plot of hemisphericscale $\sim \mathrm{N}-\mathrm{S}$ slopes vs. longitude that is characterized by a quasi-sinusoidal variation. The largest peak corresponds to Tharsis and the secondary peak is near the Tharsis antipode. Superimposed on the secondary peak is a south-sloping sig- nature associated with the Utopia depression. We test the hypothesis that the antipodal peak is related to the formation of Tharsis.

The growth of the Tharsis rise would have affected longwavelength slopes within the province and elsewhere because the re-distribution of material associated with its construction would have changed the position of Mars' COM. This COM shift would be along Tharsis' central axis and be a minimum if Tharsis formed isostatically.

Simple models can illustrate the formation of a longwavelength slope due to displacement of the COM along the axis of Tharsis, assuming instantaneous formation. For simplicity we assume end-member cases for a planet that has an average radius $R=3396 \mathrm{~km}$ and density $\rho=3530$ $\mathrm{kg} \mathrm{m}{ }^{-3}$, and ignore the presence of the core. We model Tharsis topography as a spherical cap of mean thickness $h_{1}=4 \mathrm{~km}$, angular radius $\phi_{o}=45^{\circ}$ and density $\rho_{1}=2800$ $\mathrm{kg} \mathrm{m}^{-3}$. In Model 1 we assume that Tharsis formed isostatically [Finnerty et al., 1988]. Compensation is maintained by an underlying chemical residuum layer formed in response to mantle melting that contributed to the formation of Tharsis. The residuum layer has a thickness $h_{2}$ and subtends the same angular radius as Tharsis. The change in COM

$$
y=\frac{3}{16}\left(\frac{\rho_{1}}{\rho}\right)\left(\frac{h_{1}}{R}\right)\left(h_{1}+h_{2}\right)\left(1-2 \cos \phi_{o}\right)
$$

will induce a long-baseline topographic slope on the planetary shape. Unlike the longitudinal slope of $0.036^{\circ}$ associated with the polar axis $\mathrm{COM} / \mathrm{COF}$ offset, the slope associated with the formation of Tharsis will vary with longitude. The maximum slope will be associated with Tharsis itself, and there should be another peak, $\alpha$, at the antipode. As an example we consider a 200-km thick compensating depletion layer, which has a density of $3474 \mathrm{~kg} \mathrm{~m}^{-3}$ if isostasy is maintained. The expected COM/COF offset is $35 \mathrm{~m}$, the corresponding value of $\alpha$ is $0.002^{\circ}$ over a $1000-\mathrm{km}$ baseline, and the change in surface gravity at the antipode due to the re-distribution of mass is $\sim-3 \mathrm{mGal}$. Because, Tharsis deviates from full compensation, Model 1 represents a lower limit.

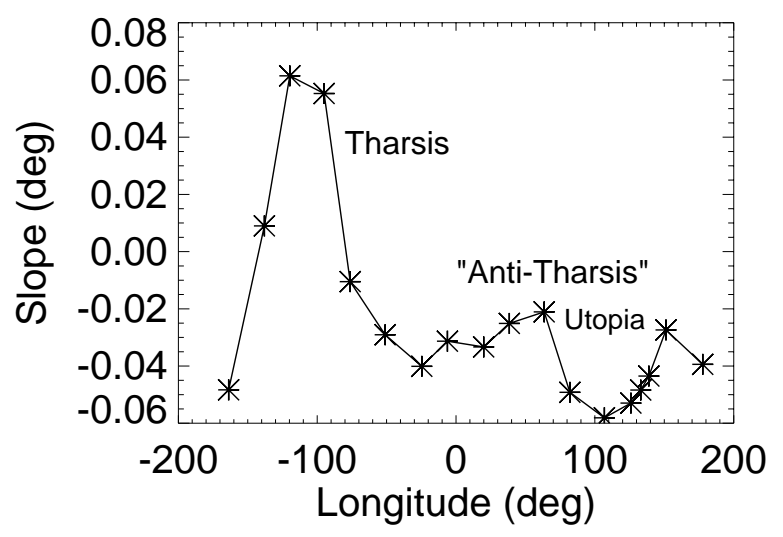

Figure 3. Hemispheric-scale slopes along MGS spacecraft groundtracks. Note the peaks in the longitudinal slope distribution associated with Tharsis and its antipode i.e. "antiTharsis". 
In Model 2 we consider a scenario in which the chemical depletion is instead distributed throughout the entire volume of Mars and Tharsis topography is largely uncompensated. Distribution of chemical depletion throughout the Martian mantle is plausible given the global-scale extent of Tharsis and the liklihood of convective mixing. The changes in the COM

$$
y=\frac{3}{8}\left(\frac{\rho_{1}}{\rho}\right) h_{1}\left(1+\frac{h_{1}}{R}\right)\left(1-2 \cos \phi_{o}\right) .
$$

$(1.2 \mathrm{~km}), \alpha\left(0.068^{\circ}\right)$, and $\Delta g_{\text {antipode }}(-260 \mathrm{mGal})$, are considerably larger than in Model 1.

The observed slopes cannot be rigorously compared to model predictions because of the simplicity of the analysis and the present uncertainty in the compensation state of Mars. However, the peak in the slope distribution at the Tharsis antipode suppports a relationship to the formation of Tharsis. We note that the predicted slopes from the end-member models bound the residual observed antipodal slope. Further analysis will focus on refining the role of Tharsis compensation on slope distributions, and on attempts to isolate other potential long-wavelength influences on planetary shape (e.g. mantle convection). These preliminary observations illustrate that the accuracy of the MOLA observations will enable recognition of subtle signals that can ultimately be used to clarify the structure, shape, uplift and subsidence history of Mars.

Acknowledgments. This study was supported by the Mars Global Surveyor Project. We acknowledge the engineers who have made these observations possible, and thank W. Kiefer and S. Smrekar for constructive reviews.

\section{References}

Albee, A. A., F. D. Palluconi, and R. E. Arvidson, Mars Global Surveyor Mission: Overview and status, Science, 279, 1671$1672,1998$.

Aharonson, O., M. T. Zuber, G.A. Neumann, and J. W. Head III, Mars: Northern hemisphere slope distributions and stochastic properties of topography, Geophys. Res. Lett., this issue, 1998.

Bills, B. G., and A. J. Ferrari, Mars topography harmonics and geophysical implications, J. Geophys. Res., 83, 3497-3508, 1978.

Finnerty, A. A., R. J. Phillips, and W. B. Banerdt, Igneous processes and closed system evolution of the Tharsis region, Mars, J. Geophys. Res., 93, 10,225-10,235, 1988.
Folkner, W. M., et al., Interior structure and seasonal mass redistribution of Mars from radio tracking of Mars Pathfinder, Science, 278, 1749-1752, 1997.

Kaula, W. M., Theory of Satellite Geodesy, 124 pp. Blaidsell, Waltham, Mass., 1966.

Kiefer, W. S., B. G. Bills, and R. S. Nerem, An inversion of gravity and topography for mantle and crustal structure on Mars, $J$. Geophys. Res., 94, 2753-2759, 1989.

McGill, G.E., Buried topography of Utopia, Mars: Persistence of a giant impact depression, J. Geophys. Res., 101, 9239-9252, 1989.

Parker, T. J., et al., Coastal geomorphology of the Martian northern plains, J. Geophys. Res., 98, 11,061-11,078, 1993.

Smith, D. E., and M. T. Zuber, The shape of Mars and the topographic signature of the hemispheric dichotomy, Science, 271, 184-188, 1996.

Smith, D. E., F. J. Lerch, R. S. Nerem, M. T. Zuber, G. B. Patel, S. K. Fricke, and F. G. Lemoine, An improved gravity model for Mars: Goddard Mars Model-1 (GMM-1), J. Geophys. Res., 98, 20,781-20,889, 1993.

Smith, D. E., et al., Topography of the northern hemisphere of Mars from the Mars Orbiter Laser Altimeter (MOLA), Science, 279, 1686-1692, 1998.

Solomon, S. C., et al., An overview of lithospheric flexure on Mars: Implications of initial MOLA observations, Lunar Planet Sci., XXIX, 1389-1390, 1998.

Wu, S. S. C., Topographic Maps of the Polar, Western, and Eastern Regions of Mars, Scale 1:15,000,000, U.S. Geol. Survey Map I-2160, 1991.

Zuber, M. T., and D. E. Smith, Mars without Tharsis, J. Geophys. Res., 102, 28,673-28,685, 1997.

Zuber, M. T., et al., The Mars Observer Laser Altimeter investigation, J. Geophys. Res., 97, 7781-7797, 1992.

O. Aharonson and M.T. Zuber, Dept. of Earth, Atmospheric, and Planetary Sci., 54-518, MIT, Cambridge, MA 02139 (e-mail: oded@mit.edu; zuber@mit.edu)

W. B. Banerdt, Jet Propulsion Laboratory 4800 Oak Grove Drive, Pasadena, CA 91109 (e-mail: William.Banerdt@ jpl.nasa.gov)

G. A. Neumann and D. E. Smith, Laboratory for Terrestrial Physics, Code 920, NASA GSFC, Greenbelt, MD 20771 (e-mail: dsmith@tharsis.gsfc.nasa.gov; neumann@tharsis.gsfc.nasa.gov)

R. J. Phillips, Dept. of Earth and Planetary Sci., Washington Univ., St. Louis, MO 63130 (e-mail: phillips@wustite.wustl.edu) S. C. Solomon, Dept. of Terrestrial Magnetism, Carnegie Inst. of Washington, 5241 Broad Branch Road, N.W., Washington, DC 200015 (e-mail: scs@dtm.ciw.edu)

(Received August 3, 1998; revised September 18, 1998; accepted September 23, 1998.) 